\title{
D-dimer/troponin ratio in the differential diag- nosis of acute pulmonary embolism from non-ST elevation myocardial infarction
}

Jong Yoon $\mathrm{Kim}^{1}$, Kye Hun Kim ${ }^{1,2}$, Jae Yeong $\mathrm{Cho}^{1,2}$, Doo Sun $\mathrm{Sim}^{1}$, Hyun Ju Yoon ${ }^{1,2}$, Nam Sik Yoon ${ }^{1}$, Young Joon Hong ${ }^{1}$, Hyung Wook Park ${ }^{1}$, Ju Han Kim ${ }^{1}$, Youngkeun Ahn ${ }^{1}$ Myung Ho Jeong ${ }^{1}$, Jeong Gwan Cho ${ }^{1}$, and Jong Chun Park ${ }^{1}$

${ }^{1}$ Department of Cardiovascular Medicine, ${ }^{2}$ Translational Research Center on Aging, Chonnam National University Hospital, Gwangju, Korea

Received: April 25, 2018

Revised : May 29, 2018

Accepted: June 21, 2018

\section{Correspondence to}

Kye Hun Kim, M.D.

Department of Cardiovascular

Medicine, Chonnam National

University Hospital, 42 Jebong-ro,

Dong-gu, Gwangju 61469, Korea

Tel: $+82-62-220-6266$

Fax: +82-62-223-3105

E-mail: christiankyehun@hanmail.net
Background/Aims: The aim of this study was to investigate useful cardiac biomarkers in the differential diagnosis of acute pulmonary embolism (APE) with troponin elevation from acute non-ST elevation myocardial infarction (NSTEMI). Methods: A total of 771 consecutive NSTEMI patients with D-dimer measurements and 90 patients with troponin-I (TnI) elevation out of 233 APE patients were enrolled, and cardiac biomarkers were compared.

Results: D-dimer elevation was noted in 382 patients with NSTEMI (49.5\%), and TnI elevation was noted 90 out of 233 APE patients (38.6\%). Unnecessary coronary angiography was performed in 10 patients (11.1\%) among 90 APE patients with TnI elevation. D-dimer was significantly elevated in APE than in NSTEMI (9.9 \pm $11.6 \mathrm{mg} / \mathrm{L}$ vs. $1.8 \pm 4.3 \mathrm{mg} / \mathrm{L}, p<0.001$ ), whereas TnI was significantly elevated in NSTEMI $(22.4 \pm 41.5 \mathrm{ng} / \mathrm{mL}$ vs. $0.7 \pm 1.4 \mathrm{ng} / \mathrm{mL}, p<0.001)$. D-dimer $/ \mathrm{TnI}$ ratio was significantly higher in APE than in NSTEMI (50.6 \pm 85.3 vs. $1.6 \pm 5.7, p<0.001)$. On receiver operation characteristic curve analysis, the optimal cut-off value for differentiating APE from NSTEMI was $1.12 \mathrm{mg} / \mathrm{L}$ for D-dimer (sensitivity 81.1\%, specificity $70.2 \%$ ), $0.72 \mathrm{ng} / \mathrm{mL}$ for $\mathrm{TnI}$ (sensitivity $80.6 \%$, specificity $78.9 \%$ ), and 1.82 for D-dimer/TnI ratio (sensitivity $93.3 \%$, specificity $86.6 \%$ ).

Conclusions: D-dimer/TnI ratio would be a simple and useful parameter for differentiating APE with cardiac troponin elevation from NSTEMI. Optimal cardiovascular imaging to identify APE should be considered in patients with D-dimer/ TnI ratio $>1.82$ before performing coronary angiography to avoid unnecessary invasive procedure.

Keywords: Pulmonary embolism; Myocardial infarction; Biomarkers

\section{INTRODUCTION}

Acute chest pain and/or dyspnea are frequently encountered clinical symptoms at emergency room, and acute pulmonary embolism (APE) is a major cause of mortality and morbidity among these patients [1]. To select appro- priate therapeutic strategy or to minimize the mortality and morbidity, the prompt and accurate identification of the life-threatening diseases such as APE or acute coronary syndromes (ACS) would be essential. Because substantial numbers of patients with APE present with dyspnea, chest pain, and electrocardiographic changes, as 
well as elevation of cardiac biomarkers which are quietly mimic for those of ACS $[2,3]$, the differential diagnosis between APE with troponin elevation and ACS without ST elevation on electrocardiography such as acute nonST elevation myocardial infarction (NSTEMI) is often difficult.

Several cardiac biomarkers have been proposed and used for rapid evaluation and differential diagnosis of the patients with acute chest pain or dyspnea at emergency setting $[4,5]$. D-dimer, a degradation product of cross-linked fibrin, is the most useful biomarker in diagnosing or ruling out APE with its proven high sensitivity [2,6-8]. However, many other conditions including acute NSTEMI may result in the elevation of serum D-dimer level, because serum D-dimer level is specific for fibrin, not for APE [9-11]. Cardiac troponins are most useful biomarkers in the diagnosis of acute NSTEMI, but other life-threatening conditions, such as APE and aortic dissection, may also result in the elevation of troponin levels [12,13]. For these reasons, the differential diagnosis between APE and acute NSTEMI becomes more difficult, and thus unnecessary coronary angiography (CAG) is performed in some cases of APE.

Therefore, the aims of the present study were to investigate useful cardiac biomarkers in the differential diagnosis of APE from acute NSTEMI and to investigate the incidence of unnecessary CAG in patients with APE.

\section{METHODS}

\section{Study design and subjects}

The present study is a single center retrospective observational study, and the study protocol was approved by the Institutional Review Board of Chonnam National University Hospital (IRB No. 2015-05-092). The waiver of informed consent is applied for the retrospective analysis study in our institution.

From 2012 to 2013, a total of 233 consecutive patients with APE and 828 patients with acute NSTEMI were identified. After exclusion of 143 patients without troponin-I (TnI) elevation, a total of 90 APE patients (68.2 \pm 15.4 years, 29 males) with TnI elevation were enrolled. After exclusion of 57 patients without measurement of D-dimer, a total of 771 patients $(66.3 \pm 12.9$ years, 521 males) with acute NSTEMI were finally enrolled in the present study.

\section{Diagnosis of APE and NSTEMI}

APE was confirmed by demonstrating the presence of contrast filling defects in pulmonary arteries on helical computed tomographic pulmonary angiography (CTPA). CTPA images were obtained with a commercially available helical CT scanner (Sensation Cardiac 64, Siemens Medical Systems, Erlangen, Germany; Light Speed QX/I, GE Medical systems, Milwaukee, WI, USA) according to the standardized APE protocol of our institution. The APE protocol requires injection of contrast material at rates of $4 \mathrm{~mL} / \mathrm{sec}$, total 70 to $80 \mathrm{~mL}$ of contrast agents, a section thickness of $3 \mathrm{~mm}$ or less, and the use of bolus tracking software for optimal opacification of pulmonary arteries.

Acute myocardial infarction (MI) was diagnosed according to the consensus document of the Joint European Society of Cardiology/American College of Cardiology Foundation/American Heart Association/World Heart Federation Task Force for the Universal Definition of Myocardial Infarction [14]. Acute NSTEMI was defined as acute MI without ST segment elevation on electrocardiography at presentation. Infarct-related arteries were identified using a combination of electrocardiographic findings, left ventricular (LV) wall motion abnormalities on echocardiography, and coronary angiographic findings.

\section{Echocardiographic examination}

Echocardiographic examination was performed as soon as possible after diagnosis of APE by CTPA for the risk stratification or clinical decision making of therapeutic strategy. Echocardiographic demonstration of right ventricular (RV) free wall hypokinesia or akinesia in patients with APE was considered as RV dysfunction. RV enlargement, defined by the ratio of RV to LV size more than 1 in apical 4 chamber view without obvious causes, accompanied by pulmonary hypertension in patients with APE was also considered as RV dysfunction in the present study [15].

\section{Measurement of biomarkers}

D-dimer, TnI, N-terminal pro-B-type natriuretic peptide (NT-proBNP), C-reactive protein (CRP) were measured as cardiac biomarkers. D-dimer was measured 
with immunoturbidimetric assay using CA-7000 system (Sysmex, Kobe, Japan), and the reference value is $<0.5$ $\mathrm{mg} / \mathrm{L}$. TnI was measured using electrochemiluminescence sandwich immunoassay method using Dimension RxL-Max (Siemens), and the reference value is < $0.05 \mathrm{ng} / \mathrm{mL}$. NT-proBNP was measured using an electrochemiluminescence sandwich immunoassay method for NT-proBNP with an Elecsys 2010 analyzer (Roche Diagnostics, Mannheim, Germany). The analytic range of the NT-proBNP assay extends from 5 to $35,000 \mathrm{pg} / \mathrm{mL}$. The reference value varies according to age and gender, and $<88 \mathrm{pg} / \mathrm{mL}$ for men and $<153 \mathrm{pg} / \mathrm{mL}$ for women in our institution. High-sensitivity C-reactive protein (hsCRP) was measured by the immunoturbidimetric CRP-Latex (II) assay using an Olympus 5431 autoanalyzer (Olympus America Inc., Center Valley, PA, USA) with reference range of $<0.5 \mathrm{mg} / \mathrm{dL}$.

\section{Statistical analysis}

The SPSS for Windows version 18 (SPSS Inc., Chicago, IL, USA) was used for statistical analyses. Data were ex- pressed as mean \pm standard deviation for continuous variables and percentage for categorical data. Chi-square test was used to compare the differences of categorical values, and independent $t$ test was used to compare the differences of continuous variables between the groups. Receiver operating characteristic (ROC) curve analysis was conducted to identify the optimal cut-off value, sensitivity and specificity of biomarkers for differentiating APE from NSTEMI. A p value less than 0.05 were considered as statistically significant.

\section{RESULTS}

\section{Baseline clinical characteristics}

Baseline characteristics of the patients with APE and NSTEMI are summarized in Table 1. Female was predominant in APE, whereas male was predominant in acute NSTEMI. Dyspnea was more common presentation in APE, whereas chest pain was more common in acute NSTEMI. Blood pressures were significantly low-

Table 1. Baseline clinical characteristics of the patients

\begin{tabular}{|c|c|c|c|}
\hline Characteristic & $\operatorname{APE}(n=90)$ & $\operatorname{NSTEMI~}(\mathrm{n}=771)$ & $p$ value \\
\hline Age, yr & $68.2 \pm 15.4$ & $66.3 \pm 12.9$ & 0.201 \\
\hline Female sex & $61(67.8)$ & $250(32.4)$ & $<0.001$ \\
\hline $\mathrm{SBP}, \mathrm{mmHg}$ & $114.4 \pm 23.0$ & $126.6 \pm 23.5$ & $<0.001$ \\
\hline $\mathrm{DBP}, \mathrm{mmHg}$ & $70.2 \pm 15 \cdot 5$ & $79.1 \pm 14.9$ & $<0.001$ \\
\hline Heart rate, beats/min & $90.1 \pm 17.5$ & $81.2 \pm 18.9$ & $<0.001$ \\
\hline \multicolumn{4}{|l|}{ Clinical presentation } \\
\hline Dyspnea & $65(72.2)$ & $155(20.1)$ & $<0.001$ \\
\hline Chest pain & $22(24.4)$ & $602(78.1)$ & $<0.001$ \\
\hline Hypotension & $13(14 \cdot 4)$ & $41(5 \cdot 3)$ & 0.001 \\
\hline Symptom to ER time, hr & $40.2 \pm 30.0$ & $21.6 \pm 21.4$ & $<0.001$ \\
\hline \multicolumn{4}{|l|}{ Predisposing conditions } \\
\hline Hypertension & $46(51.1)$ & $420(54.5)$ & 0.577 \\
\hline Smoking & $20(22.2)$ & $425(55 \cdot 1)$ & $<0.001$ \\
\hline Hypercholesterolemia & $16(17.8)$ & $157(20.3)$ & 0.562 \\
\hline Diabetes & $18(20.0)$ & $229(29.7)$ & 0.064 \\
\hline Previous VTE & $7(7.8)$ & $12(1.6)$ & $<0.001$ \\
\hline Immobilization & $30(33 \cdot 3)$ & $28(3.6)$ & $<0.001$ \\
\hline Obesity & $19(21.1)$ & $137(17.8)$ & 0.436 \\
\hline
\end{tabular}

Values are presented as mean \pm SD or number (\%).

APE, acute pulmonary embolism; NSTEMI, non-ST elevation myocardial infarction; SBP, systolic blood pressure; DBP, diastolic blood pressure; ER, emergency room; VTE, venous thromboembolism. 
Table 2. Electrocardiographic findings of the patients

\begin{tabular}{|c|c|c|c|}
\hline Variable & $\operatorname{APE}(n=90)$ & $\operatorname{NSTEMI}(\mathrm{n}=771)$ & $p$ value \\
\hline $\mathrm{HR}>100$ beats/min & $28(31.1)$ & $116(15.1)$ & $<0.001$ \\
\hline Atrial fibrillation & $6(6.7)$ & $40(5.2)$ & 0.555 \\
\hline ST depression > $1 \mathrm{~mm}$ & $6(6.7)$ & $140(18.2)$ & 0.006 \\
\hline ST elevation $>1 \mathrm{~mm}$ in aVR & $26(28.9)$ & $126(16.3)$ & 0.003 \\
\hline T-wave inversion in $\mathrm{V}_{1-3}$ & $39(43 \cdot 3)$ & $183(23.7)$ & $<0.001$ \\
\hline Incomplete or complete RBBB & $16(17.8)$ & $57(7.4)$ & 0.001 \\
\hline LBBB & o & $19(2.5)$ & 0.132 \\
\hline
\end{tabular}

Values are presented as number (\%).

APE, acute pulmonary embolism; NSTEMI, non-ST elevation myocardial infarction; HR, heart rate; RBBB, right bundle branch block; LBBB, left bundle branch block.

Table 3. Echocardiographic findings of the patients

\begin{tabular}{lccc}
\hline Variable & APE $(\mathrm{n}=90)$ & NSTEMI $(\mathrm{n}=771)$ & $p$ value \\
\hline LVEDD, mm & $42.7 \pm 7.0$ & $50.5 \pm 6.5$ & $<0.001$ \\
LVESD, mm & $27.9 \pm 5.8$ & $35.2 \pm 11.0$ & $<0.001$ \\
LVEF, \% & $61.9 \pm 10.9$ & $55.0 \pm 12.6$ & $<0.001$ \\
E, cm/sec & $51.8 \pm 15.6$ & $92.4 \pm 127.4$ & $<0.001$ \\
A, cm/e & $76.5 \pm 17.8$ & $111.3 \pm 156.1$ & $<0.001$ \\
DT, msec & $204.3 \pm 100.9$ & $212.5 \pm 71.3$ & 0.554 \\
RVSP, mmHg & $56.3 \pm 17.1$ & $36.7 \pm 11.4$ & $<0.001$ \\
RV dysfunction & $76(84.4)$ & $33(4.3)$ & $<0.001$ \\
LV dysfunction & $9(10.3)$ & $226(30.5)$ & $<0.001$ \\
\hline
\end{tabular}

Values are presented as mean \pm SD or number (\%).

APE, acute pulmonary embolism; NSTEMI, non-ST segment elevation myocardial infarction; LVEDD, left ventricular end-diastolic dimension; LVESD, left ventricular end-systolic dimension; LVEF, left ventricular ejection fraction; E, early diastolic velocity of mitral inflow; A, late diastolic velocity of mitral inflow; DT, deceleration time of mitral inflow; RVSP, right ventricular systolic pressure; RV, right ventricular; LV, left ventricular.

er and the incidence of hypotension significantly higher in APE than in NSTEMI. Immobilization and previous history of venous thromboembolism were common predisposing conditions in APE, whereas smoking was common in NSTEMI.

\section{Electrocardiographic findings}

Electrocardiographic findings between the groups are summarized in Table 2. Tachycardia, ST elevation in lead aVR, T wave inversion in leads $\mathrm{V}_{1-3}$, and right bundle branch block were more common in APE, whereas ST depression was more common in NSTEMI.

\section{Echocardiographic findings}

Echocardiographic findings between of the groups are summarized in Table 3. LV end-diastolic and end-systolic dimension were significantly smaller in APE group than in NSTEMI group. LV ejection fraction was significantly lower in NSTEMI group than in APE group. RV systolic pressure was significantly higher and RV dysfunction was significantly prevalent in APE group than in NSTEMI group, whereas LV dysfunction was significantly frequent in NSTEMI group than in APE group.

\section{Cardiac biomarkers}

The comparisons of cardiac biomarkers between the groups are summarized in Table 4. D-dimer eleva- 
Table 4. Cardiac biomarkers of the patients

\begin{tabular}{lccr}
\hline Variable & APE $(\mathrm{n}=90)$ & NSTEMI $(\mathrm{n}=771)$ & \multicolumn{1}{c}{$p$ value } \\
\hline NT-proBNP, pg/mL & $4,010.8 \pm 4,073.2$ & $3,723.1 \pm 9,847.1$ & 0.784 \\
TnI, ng/mL & $0.7 \pm 1.4$ & $22.4 \pm 41.5$ & $<0.001$ \\
D-dimer, mg/L & $9.9 \pm 11.6$ & $1.8 \pm 4.3$ & $<0.001$ \\
D-dimer/TnI ratio & $50.6 \pm 85.3$ & $1.6 \pm 5.7$ & $<0.001$ \\
hsCRP, mg/dL & $3.5 \pm 4.4$ & $2.1 \pm 4.2$ & 0.114 \\
\hline
\end{tabular}

Values are presented as mean $\pm \mathrm{SD}$.

APE, acute pulmonary embolism; NSTEMI, non-ST segment elevation myocardial infarction; NT-proBNP, N-terminal pro-Btype natriuretic peptide; TnI, troponin-I; hsCRP, high-sensitivity C-reactive protein.

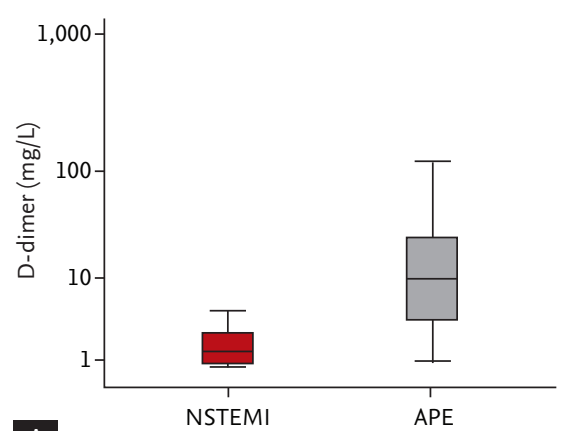

A

Figure 1. Comparisons of biomarkers between acute non-ST elevation myocardial infarction (NSTEMI) and acute pulmonary embolism (APE). (A) D-dimer, (B) troponin-I, and (C) D-dimer/troponin-I.

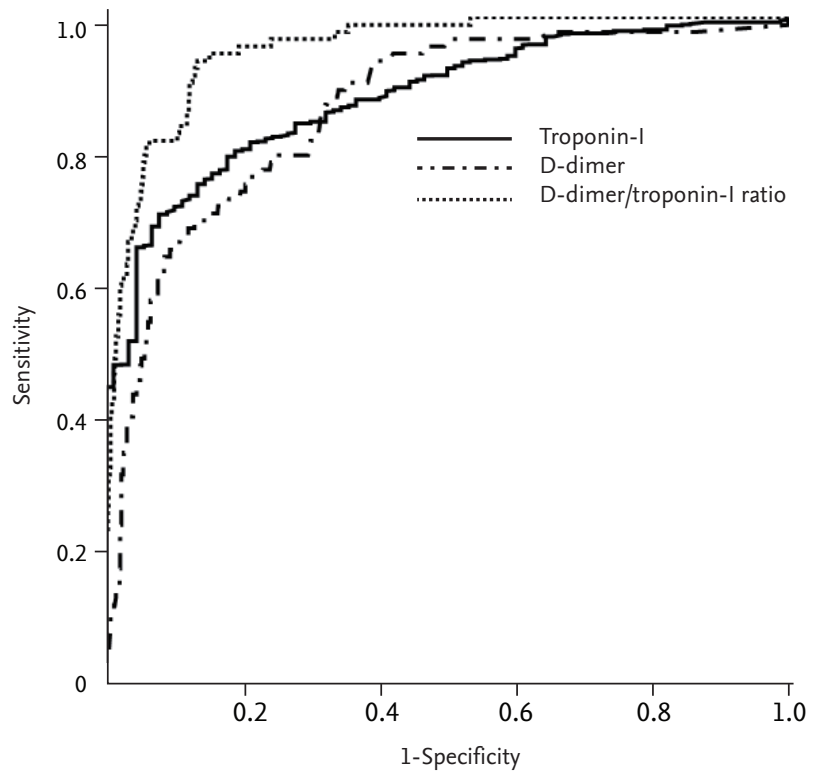

Figure 2. Receiver operating characteristic curve analysis for differential diagnosis of acute pulmonary embolism from non-ST elevation myocardial infarction. tion was noted in all patients with APE and in 382 patients with NSTEMI (49.5\%). The level of D-dimer was significantly elevated in APE group than in NSTEMI group, whereas cardiac TnI was significantly elevated in NSTEMI group than in APE group. D-dimer/TnI ratio was significantly higher in APE group than in NSTEMI group (Fig. 1). The level of NT-proBNP and hsCRP were not different between the groups.

\section{ROC curve analysis}

ROC curve analysis was performed to identify the optimal cut-off value and area under the curve (AUC) for differentiating APE from NSTEMI. The optimal cut-off value was $1.12 \mathrm{mg} / \mathrm{L}$ for D-dimer (AUC 0.860 , sensitivity $81.1 \%$, specificity $70.2 \%$ ), $0.72 \mathrm{ng} / \mathrm{mL}$ for $\mathrm{TnI}$ (AUC 0.875 , sensitivity $80.6 \%$, specificity $78.9 \%$ ), and 1.82 for D-dimer/TnI ratio (AUC 0.951, sensitivity 93.3\%, specificity $86.6 \%$ ) (Fig. 2). D-dimer/TnI ratio showed better sensitivity and specificity in the differential diagnosis between APE and NSTEMI than either D-dimer or TnI. 


\section{Unnecessary CAG and its clinical impacts}

Unnecessary CAG was performed in 10 out of 90 patients with APE (11.1\%) because initial clinical diagnosis at emergency room was acute NSTEMI. All of them had RV dysfunction on echocardiography, and four of them were hypotensive at the time of presentation. Thrombolytic therapy with recombinant tissue plasminogen activator was applied to six of them and followed by intravenous heparinization. Although all of them recovered from APE and successfully discharged, two of them experienced large puncture site hematoma after the administration of thrombolytic therapy.

\section{In-hospital mortality and biomarkers}

During hospitalization, five patients in APE (5.6\%) and 25 patients in NSTEMI (3.2\%) were died, and in-hospital mortality was not different between the groups $(p=$ $0.258)$.

Overall, the level of TnI and D-dimer and D-dimer/ TnI ratio were not different between the dead and the survived $(22.2 \pm 42.1 \mathrm{ng} / \mathrm{mL}$ vs. $20.0 \pm 39.8 \mathrm{ng} / \mathrm{mL}, p=0.780$; $4.7 \pm 8.0 \mathrm{mg} / \mathrm{L}$ vs. $2.6 \pm 6.0 \mathrm{mg} / \mathrm{L}, p=0.056 ; 5.5 \pm 8.3$ vs. $6.8 \pm$ $33.3, p=0.487$, respectively). Only the level of NT-proBNP was significantly elevated in the dead than in the survived $(8,442.0 \pm 10,652.8 \mathrm{pg} / \mathrm{mL}$ vs. 3,583.7 \pm 9,324.9 pg/mL, $p=0.005)$.

In APE group, the level of TnI and NT-proBNP were significantly elevated in the dead than in the survived $(2.2 \pm 2.5 \mathrm{ng} / \mathrm{mL}$ vs. $0.6 \pm 1.3 \mathrm{ng} / \mathrm{mL}, p=0.01 ; 8,678.4 \pm$ 4,439.0 pg/mL vs. $3,736.3 \pm 3,907.4 \mathrm{pg} / \mathrm{mL}, p=0.008$, respectively), whereas D-dimer/TnI ratio was significantly lower in the dead than in the survived ( $12.2 \pm 11.7$ vs. 52.9 $\pm 87.2, p<0.001)$. The level of D-dimer was not different between the dead and the survived $(15.9 \pm 14.8$ vs. $9.6 \pm$ $11.4 \mathrm{mg} / \mathrm{L}, p=0.237$ ).

In NSTEMI group, the level of NT-proBNP and D-dimer/TnI ratio were significantly higher in the dead than in the survived $(8,394.7 \pm 11,568.3 \mathrm{pg} / \mathrm{mL}$ vs. $3,566.5$ $\pm 9,754.6 \mathrm{pg} / \mathrm{mL}, p=0.016 ; 4.1 \pm 7.0$ vs. $1.5 \pm 5.6, p=0.024$, respectively). However, the levels of TnI and D-dimer were not different between the dead and the survived $(26.2 \pm 45.2 \mathrm{ng} / \mathrm{mL}$ vs. $22.2 \pm 41.4 \mathrm{ng} / \mathrm{mL}, p=0.668 ; 2.5 \pm 3.1$ $\mathrm{mg} / \mathrm{L}$ vs. $1.8 \pm 4.3 \mathrm{mg} / \mathrm{L}, p=0.273$, respectively).

\section{DISCUSSION}

The authors investigated useful cardiac biomarkers in the differential diagnosis of APE from acute NSTEMI and the incidence of unnecessary CAG in patients with APE, and the results of the present study demonstrated several clinically important findings. Firstly, D-dimer/ Tni ratio is a simple, but very sensitive and specific parameter for differentiating APE with troponin elevation from NSTEMI. Secondly, unnecessary CAG is performed not infrequently in patients with APE (11.1\%), because acute NSTEMI was the initial clinical diagnosis in patients with chest pain and/or dyspnea and troponin elevation. Thirdly, unnecessary CAG is associated with bleeding complications in patients with APE who require thrombolytic therapy.

Although the prompt diagnosis and adequate therapy is essential in reducing mortality and complications, APE is sometimes misdiagnosed as acute NSTEMI in emergency room because of their similarities in clinical presentation $[2,3]$. In the present study, 10 out of 90 APE patients were also initially misdiagnosed as acute NSTEMI, and thus unnecessary CAG was performed. All of them had RV dysfunction and four of them were hypotensive, and thus they can be classified as intermediate-high or high risk group of APE. Because in-hospital death is usually developed in first few hours after admission, these misdiagnoses of APE may result in increased mortality even though no deaths were developed in the present study fortunately. Furthermore, unnecessary CAG was associated with bleeding complications after thrombolytic therapy in the present study. Therefore, the authors want to investigate simple biomarker based approach to reduce the misdiagnosis of APE from acute NSTEMI.

The level of D-dimer can be elevated in a wide variety of clinical conditions associated with simultaneous activation of coagulation and fibrinolysis, and these include inflammation, bleeding, trauma, surgery, and necrosis [3,9-11]. In the present study, 382 patients (49.5\%) with acute NSTEMI also showed D-dimer elevation. Because acute NSTEMI is also a condition of acute thrombosis, it is expected that D-dimer level is elevated in patients with acute NSTEMI. For these reasons, the positive predictive value of $\mathrm{D}$-dimer testing is low, and thus $\mathrm{D}$-dimer testing is not useful for the confirm diagnosis of APE [16]. 
The major clinical usefulness of D-dimer testing is that a normal D-dimer level can usually exclude the possibility of APE because of its high negative predictive value. Despite of low positive predictive value, some studies have suggested that D-dimer testing is useful in the differential diagnosis of APE from acute MI. In the study of Sakamoto et al. [11], D-dimer level $>5.0 \mathrm{~g} / \mathrm{mL}$ is helpful for differentiating APE from acute MI with acceptable specificity of $90 \%$, but the sensitivity of D-dimer testing was only $68 \%$. The authors also evaluated the usefulness of D-dimer testing in differentiating APE from acute NSTEMI. The optimal cut-off value of D-dimer for differentiating APE from NSTEMI was $1.12 \mathrm{mg} / \mathrm{L}$, and the sensitivity was $81.1 \%$, and specificity was $70.2 \%$ in the present study. As in the study of Sakamoto et al. [11], our study also demonstrated that D-dimer testing has limitation in the differential diagnosis of APE from acute NSTEMI because of its low sensitivity.

Cardiac troponins are most useful biomarkers in the diagnosis of acute MI [12,13], but APE may also results in the elevation of troponin levels, especially in patients with RV dysfunction on imaging study or severe APE $[3,17,18]$. In the present study, TnI elevation was observed in 90 out of $233 \mathrm{APE}$ patients (38.6\%), and TnI elevation in APE was associated with RV dysfunction on echocardiography; RV dysfunction was demonstrated 77 (84.4\%) out of 90 APE patients with TnI elevation. Although the role of cardiac troponins in identifying high risk group of APE has been well evaluated, the role of troponin testing in differential diagnosis of APE from acute NSTEMI has never been studied. In the present study, the optimal cut-off value of TnI for differentiating APE from acute NSTEMI of TnI was $0.72 \mathrm{ng} / \mathrm{mL}$. The level of TnI $<0.72 \mathrm{ng} / \mathrm{mL}$ usually favors the diagnosis of APE rather than acute NSTEMI with the sensitivity of $80.6 \%$ and specificity of $78.9 \%$. To the best of our knowledge, this is the first study which demonstrated the role of troponin testing in the differential diagnosis of APE with troponin elevation from acute NSTEMI.

In the present study, the usefulness of D-dimer/TnI ratio in the differential diagnosis of APE with troponin elevation from acute NSTEMI was firstly evaluated, because the authors hypothesized that the difference of D-dimer/TnI ratio between APE and acute NSTEMI would become much greater than the difference of either D-dimer or TnI alone. D-dimer/TnI ratio was sig- nificantly higher in APE than in NSTEMI (50.6 $\pm 85.3 \mathrm{vs}$. $1.6 \pm 5.7, p<0.001$ ), and the D-dimer/TnI ratio of $>1.82$ can significantly differentiate APE with troponin elevation from acute NSTEMI (AUC 0.951, sensitivity 93.3\%, specificity $86.6 \%$ ). D-dimer/Tni ratio $>1.82$ showed better sensitivity and specificity than D-dimer or TnI alone in differentiating APE with troponin elevation from acute NSTEMI. From these results, the authors propose that optimal cardiovascular imaging for the identification of APE should be considered in patients with acute chest pain or dyspnea and D-dimer/TnI ratio $>1.82$ before considering acute NSTEMI and performing CAG. This simple approach may avoid the risk of unnecessary invasive procedure.

NT-proBNP is a useful cardiac biomarker for the differential diagnosis of acute dyspnea whether it is cardiac or non-cardiac origin [19]. However, the level of NT-proBNP was not different between APE with troponin elevation and acute NSTEMI in the present study. Because both APE with troponin elevation and acute NSTEMI are acutely stressful conditions for heart and natriuretic peptide is released from cardiac myocytes due to hemodynamic stress, the level of NT-proBNP may not be significantly different in both conditions. Although NT-proBNP seemed not to be an useful biomarker in the differential diagnosis of APE from NSTEMI, recent meta-analyses confirmed that NT-proBNP is a useful prognostic marker for APE [20,21].

The present study has several limitations. First, the study was a single-center, retrospective observational study, and thus the present study has all limitations of retrospective study including selection bias. Second, although the blood samplings for cardiac biomarkers were taken as soon as possible after hospitalization, the timing of blood samplings might be different among the patients, and the results of biomarker levels might be affected. Also, the time difference from symptom onset to hospitalization of each patient was not considered in the present study, but it may also affect the results of biomarker level. Third, chest CTA was not performed in patients with acute NSTEMI, and thus the presence of combined APE cannot be excluded.

Despite of these limitations, the result of the present study demonstrated that D-dimer/TnI ratio is a simple, sensitive, and specific parameter for distinguishing APE from NSTEMI. Optimal cardiovascular imaging 
to identify APE should be considered in patients with acute dyspnea or chest pain and D-dimer/TnI ratio > 1.82 before considering NSTEMI and performing CAG. This simple approach may be helpful in avoiding the risk of unnecessary invasive procedure and also helpful for patients to get optimal treatments without delay. Prospective studies with larger number of subjects will be needed to confirm the results of the present study.

\section{KEY MESSAGE}

1. Unnecessary coronary angiography $(\mathrm{CAG})$ is not infrequently performed in acute pulmonary embolism (APE) patients with troponin elevation because of clinical similarities with non-ST elevation myocardial infarction (NSTEMI).

2. Unnecessary CAG is associated with bleeding complications in patients with APE who require thrombolytic therapy.

3. The measurement of D-dimer/troponin-I ratio would be a simple and useful parameter for differentiating APE with cardiac troponin elevation from NSTEMI in acute clinical settings and thus would be helpful in avoiding unnecessary CAG.

\section{Conflict of interest}

No potential conflict of interest relevant to this article was reported.

\section{Acknowledgments}

This study was supported by a research grant(CRI 1390421) of Chonnam National University Hospital Biomedical Research Institute.

\section{REFERENCES}

1. Duru S, Kelesoglu A, Ardic S. Clinical update on pulmonary embolism. Arch Med Sci 2014;10:557-565.

2. Choi HS, Kim KH, Yoon HJ, et al. Usefulness of cardiac biomarkers in the prediction of right ventricular dysfunction before echocardiography in acute pulmonary embolism. J Cardiol 2012;60:508-513.

3. Konstantinides SV, Torbicki A, Agnelli G, et al. 2014 ESC guidelines on the diagnosis and management of acute pulmonary embolism. Eur Heart J 2014;35:3033-3069.

4. Skinner JS, Smeeth L, Kendall JM, Adams PC, Timmis A; Chest Pain Guideline Development Group. NICE guidance. Chest pain of recent onset: assessment and diagnosis of recent onset chest pain or discomfort of suspected cardiac origin. Heart 2010;96:974-978.

5. Stokes NR, Dietz BW, Liang JJ. Cardiopulmonary laboratory biomarkers in the evaluation of acute dyspnea. Open Access Emerg Med 2016;8:35-45.

6. Stein PD, Hull RD, Patel KC, et al. D-dimer for the exclusion of acute venous thrombosis and pulmonary embolism: a systematic review. Ann Intern Med 2004;140:589602 .

7. Wells PS, Anderson DR, Rodger M, et al. Excluding pulmonary embolism at the bedside without diagnostic imaging: management of patients with suspected pulmonary embolism presenting to the emergency department by using a simple clinical model and D-dimer. Ann Intern Med 2001;135:98-107.

8. van Belle A, Buller HR, Huisman MV, et al. Effectiveness of managing suspected pulmonary embolism using an algorithm combining clinical probability, D-dimer testing, and computed tomography. JAMA 2006;295:172-179.

9. Bayes-Genis A, Mateo J, Santalo M, et al. D-Dimer is an early diagnostic marker of coronary ischemia in patients with chest pain. Am Heart J 2000;140:379-384.

10. Chang SS, Lee SH, Wu JY, et al. Evaluation of the value of rapid D-dimer test in conjunction with cardiac troponin I test for early risk stratification of myocardial infarction. J Thromb Thrombolysis 2010;30:472-478.

11. Sakamoto K, Yamamoto Y, Okamatsu H, Okabe M. D-dimer is helpful for differentiating acute aortic dissection and acute pulmonary embolism from acute myocardial infarction. Hellenic J Cardiol 2011;52:123-127.

12. Amsterdam EA, Wenger NK, Brindis RG, et al. 2014 AHA/ ACC guideline for the management of patients with non-ST-elevation acute coronary syndromes: executive summary: a report of the American College of Cardiology/American Heart Association Task Force on Practice Guidelines. Circulation 2014;130:2354-2394.

13. Roffi M, Patrono C, Collet JP, et al. 2015 ESC guidelines for the management of acute coronary syndromes in patients presenting without persistent ST-segment elevation: task force for the management of acute coronary syndromes in patients presenting without persistent 
ST-segment elevation of the European Society of Cardiology (ESC). Eur Heart J 2016;37:267-315.

14. Thygesen K, Alpert JS, Jaffe AS, et al. Third universal definition of myocardial infarction. J Am Coll Cardiol 2012;60:1581-1598.

15. Kline JA, Hernandez-Nino J, Rose GA, Norton HJ, Camargo CA Jr. Surrogate markers for adverse outcomes in normotensive patients with pulmonary embolism. Crit Care Med 2006;34:2773-2780.

16. Hahne K, Lebiedz P, Breuckmann F. Impact of D-dimers on the differential diagnosis of acute chest pain: current aspects besides the widely known. Clin Med Insights Cardiol 2014;8(Suppl 2):1-4.

17. Meyer T, Binder L, Hruska N, Luthe H, Buchwald AB. Cardiac troponin I elevation in acute pulmonary embolism is associated with right ventricular dysfunction. J Am Coll Cardiol 2000;36:1632-1636.
18. Kilinc G, Dogan OT, Berk S, Epozturk K, Ozsahin SL, Akkurt I. Significance of serum cardiac troponin I levels in pulmonary embolism. J Thorac Dis 2012;4:588-593.

19. Ray P, Delerme S, Jourdain P, Chenevier-Gobeaux C. Differential diagnosis of acute dyspnea: the value of $B$ natriuretic peptides in the emergency department. QJM 2008;101:831-843.

20. Klok FA, Mos IC, Huisman MV. Brain-type natriuretic peptide levels in the prediction of adverse outcome in patients with pulmonary embolism: a systematic review and meta-analysis. Am J Respir Crit Care Med 2008;178:425430.

21. Sanchez O, Trinquart L, Colombet I, et al. Prognostic value of right ventricular dysfunction in patients with haemodynamically stable pulmonary embolism: a systematic review. Eur Heart J 2008;29:1569-1577. 